\title{
Computational stochastic statics of an uncertain curved structure with geometrical nonlinearity in three-dimensional elasticity.
}

\author{
Capiez-Lernout, E., Soize, C. and Mignolet, M.P.
}

Received: date / Accepted: date

\begin{abstract}
A methodology for analyzing the large static deformations of geometrically nonlinear structural systems in presence of both system parameters uncertainties and model uncertainties is presented. It is carried out in the context of the identification of stochastic nonlinear reduced-order computational models using simulated experiments. This methodology requires the knowledge of a reference calculation issued from the mean nonlinear computational model in order to determine the POD basis used for the mean nonlinear reduced-order computational model. The construction of such mean reduced-order nonlinear computational model is explicitly carried out in the context of three-dimensional solid finite elements. It allows the stochastic nonlinear reduced-order computational model to be constructed in any general case with the nonparametric probabilistic approach. A numerical example is then presented for a curved beam in which the various steps are presented in details.
\end{abstract}

Keywords stochastic $\cdot$ numerical methods $\cdot$ large deformation $\cdot$ post-buckling $\cdot$ finite element

E. Capiez-Lernout

Université Paris-Est, Modélisation et Simulation Multi Échelle, MSME UMR 8208 CNRS, 5 bd Descartes, 77455 Marne-la-Vallée Cedex 02, France

Tel.: +33-160957798

Fax: +33-160957799

E-mail: evangeline.capiezlernout@ univ-paris-est.fr

C. Soize

Université Paris-Est, Modélisation et Simulation Multi Échelle, MSME UMR 8208 CNRS, 5 bd Descartes, 77455 Marne-la-Vallée Cedex 02, France

M.P. Mignolet

SEMTE, Faculties of Mechanical and Aerospace Engineering, Arizona State University, Tempe, Arizona 85287-6106, USA

\section{Introduction}

A recent challenge in structural mechanics is to have advanced numerical methodologies for the construction of robust computational models in order to efficiently predict the mechanical behavior of structures. In numerous industrial applications, the effects of geometrical nonlinearities induced by large strains and by large displacements have to be taken into account in the numerical modeling. For instance, such nonlinear mechanical behavior is exhibited in aeronautics for the case of helicopter rotating blades [12,21] or in automotive or aerospace applications involving slender beams or thin shells $[4,8,6]$. In the context of complex structures, large finite element computational models are however needed. Then, given the numerical difficulties inherent to the complexity of such computational models, some recent investigations have focused on the construction of reduced order models in this nonlinear context $[13,5]$. In particular, the STEP procedure [11,9] has been developed in order to explicitly construct all linear, quadratic and cubic stiffness terms related to reduced nonlinear models. The methodology is based on the smart use of a standard commercial finite element code for which no further numerical development is needed. It only requires a series of straightforward nonlinear numerical calculations with judicious prescribed displacements taken as a linear combination of given basis vectors.

Moreover, deterministic nonlinear computational models are in general not sufficient to accurately predict the mechanical response of such complex structures. Uncertainties have then to be taken into account in the computational models by using probabilistic models as soon as the probability theory can be used. Let us recall that there are two classes of uncertainties: (1) the system-parameter uncertainties are due to the variability of the parameters of the computational model, (2) the model uncertainties, induced by modeling er- 
rors, are the features of the mechanical system not captured by the computational model, e.g. the introduction of reduced kinematics in the numerical modeling. The parametric probabilistic approach is particularly adapted to take into account system-parameter uncertainties as shown in $[14,15]$ in the context of post-buckling of cylindrical shells. This last decade, the nonparametric probabilistic approach has been developed to allow the consideration of both model uncertainties and system-parameter uncertainties. It has been introduced in $[17,19]$ for the linear case and has been extended more recently in $[9,10]$ for linearly elastic but geometrically nonlinear problems. In the present paper, a direct procedure is proposed for the explicit construction of stochastic reduced-order models of uncertain geometrically nonlinear structures. It is applicable to any type of structure modeled with three-dimensional solid finite elements and with a linear constitutive equation.

The paper is organized as follows. In section 2, the equations of the geometrical nonlinear problem are written in the context of a total Lagrangian formulation. The third Section is devoted to the construction of the mean nonlinear reduced-order computational model required by the nonparametric probabilistic approach. This mean nonlinear reducedorder model is obtained using the Proper Orthogonal Decomposition (POD) method known to be particularly efficient in nonlinear static cases [13]. The POD basis is constructed with the deterministic nominal three-dimensional computational model of the structure. The mean nonlinear reduced-order model results from the projection of the weak formulation related to the nonlinear boundary value problem, on the subspace spanned by the POD basis. It is then explicitly constructed discretizing the problem using threedimensional solid finite elements. The fourth Section is devoted to the construction of the stochastic nonlinear reduced-order computational model using the nonparametric probabilistic approach, which will introduce a random matrix $[\mathcal{K}]$ adapted to the problem. Such a nonparametric probabilistic approach is based on the construction of a probability model for the random matrix $[\mathcal{K}]$ with values in the set of symmetric positive-definite matrices whose mean value is deduced from the mean nonlinear reduced-order model. The mean value of $[\mathcal{K}]$ involves the linear, quadratic, and cubic stiffness terms of the mean nonlinear reduced order model and must be symmetric and positive definite. An explicit approach is proposed here, instead of the STEP formulation, to maintain these properties. Note that the explicit construction of each contribution is required. In the fifth Section, a procedure is given for identifying the stochastic reduced-order nonlinear computational model with respect to simulated experimental responses $[20,1,2]$. Finally, the last Section deals with an application involving a curved structure in order to demonstrate the efficiency of the proposed methodology.

\section{Weak formulation of the geometric nonlinear boundary value problem}

This Section is devoted to the weak formulation of the boundary value problem in the context of linear elasticity with geometrical nonlinearities.

2.1 Description of the geometric nonlinear boundary value problem

The structure under consideration is constructed of a linear elastic material and is assumed to undergo large deformations inducing geometrical nonlinearities. Let $\Omega$ be the three-dimensional bounded domain of the physical space $\mathbb{R}^{3}$ corresponding to the reference configuration taken as a natural state without prestresses. The boundary $\partial \Omega$ is such that $\partial \Omega=\Gamma \cup \Sigma$ with $\Gamma \cap \Sigma=\emptyset$ and the external unit normal to boundary $\partial \Omega$ is denoted by $\mathbf{n}$ (see Fig. 1). The boundary part $\Gamma$ corresponds to the fixed part of the structure whereas the boundary part $\Sigma$ is submitted to an external surface force field. A total Lagrangian formulation is chosen. Consequently, the mechanical equations are written with respect to the reference configuration. Let $\mathbf{x}$ be the position of a point belonging to domain $\Omega$. The displacement field expressed with respect to the reference configuration is denoted as $\mathbf{u}(\mathbf{x})$. It should be noted that the surface force field $\mathbf{G}(\mathbf{x})$ acting on boundary $\Sigma$ and that the body force field $\mathbf{g}(\mathbf{x})$ acting on domain $\Omega$ correspond to the Lagrangian transport into the reference configuration of the physical surface force field and to the physical body force field applied on the deformed configuration.

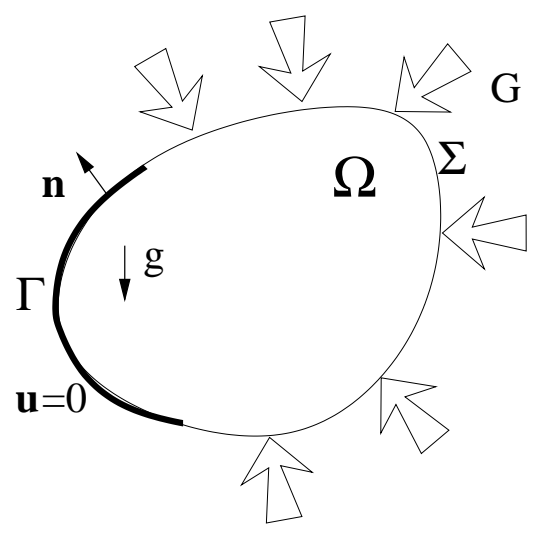

Fig. 1 Reference configuration

\subsection{Weak formulation}

The admissible space $\mathcal{C}$ defined by

$\mathcal{C}=\{\mathbf{v} \in \Omega, \mathbf{v}$ sufficiently regular, $\mathbf{v}=\mathbf{0}$ on $\Gamma\}$ 
is introduced in order to construct the weak formulation related to the geometric nonlinear boundary value problem. It then consists in finding the unknown displacement field $\mathbf{u}$ in admissible space $\mathcal{C}$ such that, for any admissible displacement field $\mathbf{v}$ in $\mathcal{C}$

$\int_{\Omega} v_{i, k} F_{i j} S_{j k} d \mathbf{x}=\int_{\Omega} v_{i} g_{i} d \mathbf{x}+\int_{\Sigma} v_{i} G_{i} d \mathbf{s}$.

In this equation, the convention of summation over repeated latin indices is used. In addition $v_{i, k}$ denotes $\frac{\partial v_{i}}{\partial x_{k}}$. In Eq. (2), $\mathbb{F}=\left\{F_{i j}\right\}_{i j}$ is the deformation gradient tensor whose components $F_{i j}$ are defined by

$F_{i j}=u_{i, j}+\delta_{i j}$,

in which $\delta_{i j}$ is the Kronecker symbol such that $\delta_{i j}=1$ if $i=j$ and $\delta_{i j}=0$ otherwise. $\mathbb{S}=\left\{S_{i j}\right\}_{i j}$ is the second Piola-Kirchoff symmetric stress tensor which is written for a linear elastic material as

$S_{i j}=a_{i j k \ell} E_{k \ell}$.

In Eq. (4), the fourth order elasticity tensor $\mathbb{a}=\left\{a_{i j k \ell}\right\}_{i j k \ell}$ satisfies the usual symmetry and positive-definiteness properties. The Green strain tensor $\mathbb{E}=\left\{E_{i j}\right\}_{i j}$ is then written as the sum of linear and nonlinear terms such that

$E_{i j}=\varepsilon_{i j}+\eta_{i j}$,

in which

$\varepsilon_{i j}=\frac{1}{2}\left(u_{i, j}+u_{j, i}\right) \quad$ and $\quad \eta_{i j}=\frac{1}{2} u_{s, i} u_{s, j}$.

\subsection{Definition of the multi-linear forms}

The weak formulation defined by Eq. (2) is then rewritten in order to distinguish the linear part from both non-linear quadratic and cubic parts of the equation. The following multi-linear forms are then introduced by writing Eq. (2) such that

$k^{(1)}(\mathbf{u}, \mathbf{v})+k^{(2)}(\mathbf{u}, \mathbf{u}, \mathbf{v})+k^{(3)}(\mathbf{u}, \mathbf{u}, \mathbf{u}, \mathbf{v})=f(\mathbf{v})$

in which the linear form $f(\mathbf{v})$ and the multi-linear forms $k^{(1)}(\mathbf{u}, \mathbf{v}), k^{(2)}(\mathbf{u}, \mathbf{u}, \mathbf{v})$ and $k^{(3)}(\mathbf{u}, \mathbf{u}, \mathbf{u}, \mathbf{v})$ are defined for all $\mathbf{u}$ and all $\mathbf{v}$ in $\mathcal{C}$ by

$$
\begin{aligned}
& f(\mathbf{v})=\int_{\Omega} v_{i} g_{i} d \mathbf{x}+\int_{\Sigma} v_{i} G_{i} d \mathbf{s} \\
& k^{(1)}(\mathbf{u}, \mathbf{v})=\int_{\Omega} a_{j k \ell m} \varepsilon_{\ell m}(\mathbf{u}) \varepsilon_{j k}(\mathbf{v}) d \mathbf{x} \\
& k^{(2)}(\mathbf{u}, \mathbf{u}, \mathbf{v})=\int_{\Omega} a_{j k \ell m} \eta_{\ell m}(\mathbf{u}) \varepsilon_{j k}(\mathbf{v}) d \mathbf{x}+ \\
& \int_{\Omega} a_{j k \ell m} u_{s, j} v_{s, k} \varepsilon_{\ell m}(\mathbf{u}) d \mathbf{x} \\
& k^{(3)}(\mathbf{u}, \mathbf{u}, \mathbf{u}, \mathbf{v})=\int_{\Omega} a_{j k \ell m} u_{s, j} v_{s, k} \eta_{\ell m}(\mathbf{u}) d \mathbf{x}
\end{aligned}
$$

\section{Construction of the mean nonlinear reduced-order computational model}

This Section focuses on the construction of the mean nonlinear reduced-order computational model. First, the equation yielding the mean nonlinear reduced-order model is written for any given projection basis. In such equation, it should be noted that the linear, quadratic and cubic stiffness contributions have particular symmetry properties which can directly be related to the elasticity tensor properties. The construction of the mean nonlinear reduced-order model is then carried out in two main steps : the numerical construction of an adapted projection basis and the explicit construction of each linear, quadratic and cubic stiffness contribution. The second paragraph is devoted to the construction of the projection basis using the Proper Orthogonal Decomposition method (POD method). The choice of such POD basis is known to be particularly adapted in the context of large finite elements systems $[16,13]$. Its construction requires the knowledge of the nonlinear static response, which is calculated from the mean nonlinear model using a finite element code. The POD basis is then defined as the eigenvectors of the spatial correlation matrix associated with the nonlinear static response. Its numerical construction is briefly recalled in the context of a large finite element model [13]. Finally, the mean nonlinear reduced-order model is explicitly determined in the context of three-dimensional solid finite elements. In particular, the integrals describing each linear, quadratic and cubic stiffness contribution are explicitly calculated using the POD basis and using the symmetry properties of the elasticity tensor.

\subsection{Equations for the mean nonlinear reduced-order computational model}

Let $\varphi^{\alpha}(\mathbf{x}), \alpha=\{1, \ldots, N\}$, be a given set of basis functions such that

$\mathbf{u}(\mathbf{x})=\sum_{\beta=1}^{N} \varphi^{\beta}(\mathbf{x}) q_{\beta}$,

in which $\mathbf{q}=\left(q_{1}, \ldots, q_{N}\right)$ is the $\mathbb{R}^{N}$-vector of the generalized coordinates. Let $\mathbf{v}(\mathbf{x})$ be a test function such that

$\mathbf{v}(\mathbf{x})=\varphi_{\alpha}(\mathbf{x}) q_{\alpha}$

Substituting Eq.(13) into Eq.(7) yields the following nonlinear equations

$\mathcal{K}_{\alpha \beta}^{(1)} q_{\beta}+\mathcal{K}_{\alpha \beta \gamma}^{(2)} q_{\beta} q_{\gamma}+\mathcal{K}_{\alpha \beta \gamma \delta}^{(3)} q_{\beta} q_{\gamma} q_{\delta}=\mathcal{F}_{\alpha}$,

in which

$\mathcal{K}_{\alpha \beta}^{(1)}=\int_{\Omega} a_{j k \ell m} \varphi_{j, k}^{\alpha} \varphi_{\ell, m}^{\beta} d \mathbf{x}$ 
$\mathcal{K}_{\alpha \beta \gamma}^{(2)}=\frac{1}{2}\left(\widehat{\mathcal{K}}_{\alpha \beta \gamma}^{(2)}+\widehat{\mathcal{K}}_{\beta \gamma \alpha}^{(2)}+\widehat{\mathcal{K}}_{\gamma \alpha \beta}^{(2)}\right)$

$\widehat{\mathcal{K}}_{\alpha \beta \gamma}^{(2)}=\int_{\Omega} a_{j k \ell m} \varphi_{j, k}^{\alpha} \varphi_{s, \ell}^{\beta} \varphi_{s, m}^{\gamma} d \mathbf{x}$

$\mathcal{K}_{\alpha \beta \gamma \delta}^{(3)}=\int_{\Omega} a_{j k \ell m} \varphi_{r, j}^{\alpha} \varphi_{r, k}^{\beta} \varphi_{s, \ell}^{\gamma} \varphi_{s, m}^{\delta} d \mathbf{x}$

$\mathcal{F}_{\alpha}=\int_{\Omega} g_{i} \varphi_{i}^{\alpha} d \mathbf{x}+\int_{\Sigma} G_{i} \varphi_{i}^{\alpha} d \mathbf{s}$.

It can easily be seen that the symmetry properties of the fourth-order elasticity tensor yield the following properties

$$
\begin{aligned}
\mathcal{K}_{\alpha \beta}^{(1)} & =\mathcal{K}_{\beta \alpha}^{(1)}, \\
\widehat{\mathcal{K}}_{\alpha \beta \gamma}^{(2)} & =\widehat{\mathcal{K}}_{\alpha \gamma \beta}^{(2)}, \\
\mathcal{K}_{\alpha \beta \gamma}^{(2)} & =\mathcal{K}_{\beta \gamma \alpha}^{(2)}=\mathcal{K}_{\gamma \alpha \beta}^{(2)}, \\
\mathcal{K}_{\alpha \beta \gamma \delta}^{(3)} & =\mathcal{K}_{\alpha \beta \delta \gamma}^{(3)}=\mathcal{K}_{\beta \alpha \gamma \delta}^{(3)}=\mathcal{K}_{\gamma \delta \alpha \beta}^{(3)} .
\end{aligned}
$$

Moreover, using the positive-definite property of the fourthorder elasticity tensor, it can be shown that tensors $\mathcal{K}_{\alpha \beta}^{(1)}$ and $\mathcal{K}_{\alpha \beta \delta \gamma}^{(3)}$ are positive definite.

3.2 Numerical construction of the basis using Proper Orthogonal Decomposition

The set of basis vectors used for constructing the mean reduced-order nonlinear computational model is obtained with the Proper Orthogonal Decomposition (POD) method which is known to be efficient for nonlinear static cases. The construction of such a basis requires a set of displacement fields solutions of the mean nonlinear computational model. Indeed such basis is defined by the eigenvalue problem of the spatial correlation operator related to this displacement field. It should be noted that the POD basis does not only depend on the operators of the computational model but also strongly depends on the external loads applied to the structure. Below, the numerical construction of the POD basis is summarized in the context of the finite element method. The finite element discretization of Eq. (7) can be written as

$\left[K^{(1)}\right] \boldsymbol{u}+\mathbb{F}^{N L}(u)=\mathbb{F}$,

in which the $\mathbb{R}^{n}$-vector $u$ is the vector of the unknown displacements. In Eq. (24), the $(n \times n)$ symmetric positivedefinite matrix $\left[K^{(1)}\right]$ is the linear finite element stiffness matrix, the $\mathbb{R}^{n}$-vector $\mathbb{E}^{N L}(u)$ is the vector of the restoring forces induced by the geometrical nonlinear effects and the $\mathbb{R}^{n}$-vector $\mathbb{E}$ is the vector of the external applied loads. It should be noted that there are specific numerical algorithms for solving this nonlinear equation (see for instance [7]) which are particularly efficient as the curvature of the nonlinear response changes (see for instance [3] for algorithms based on arc-length methods or [22] for algorithms based on asymptotic methods).
Let $s_{j} \in[0,1], j \in\{1, \ldots, p\}$ with $s_{j}<s_{j+1}$ be the scalar denoting the incremental weight number $j$ of the external load vector $\mathbb{f}$. The $(n \times p)$ real matrix $[V]$ is then introduced as

$$
\begin{aligned}
& {[V]_{i j}=\mathbb{u}_{i}\left(s_{j}\right) \sqrt{\Delta s_{j}}, \Delta s_{j}=s_{j}-s_{j-1},} \\
& \text { with } s_{0}=0 .
\end{aligned}
$$

The spatial correlation matrix related to the nonlinear response $\backsim\left(s_{j}\right)$ is the symmetric positive $(n \times n)$ real matrix $[A]$ such that

$$
[A]=[V][V]^{T} .
$$

The POD basis is then obtained by solving the following eigenvalue problem

$[A][\Phi]=[\Phi][\Lambda]$,

in which $[\Lambda]$ is the diagonal matrix whose components are the eigenvalues ordered by decreasing values and where $[\Phi]$ is the eigenvector matrix whose columns are the POD basis vectors. It should be noted that this numerical construction cannot be carried out if the dimension $n$ is large. The following methodology introduced by $[16,13]$ is used instead for large computational models. The singular value decomposition of the matrix $[V]$ is written as

$[V]=[B][S][C]$

in which $[S]=[\Lambda]^{1 / 2}$ and where the columns of the $(n \times n)$ and $(p \times p)$ real matrices $[B]$ and $[C]$ are the left and right singular vectors related to the corresponding singular values. Let $\left[B^{N}\right]$ be the $(n \times N)$ matrix issued from the column truncation of matrix $[B]$ with respect to the $N$ largest singular values. The matrix $\left[B^{N}\right]$ can be easily computed. The $(N \times N)$ symmetric positive-definite real matrix $\left[\mathcal{A}^{N}\right]$ is then introduced as

$\left[\mathcal{A}^{N}\right]=\left[W^{N}\right]\left[W^{N}\right]^{T}$, with $\left[W^{N}\right]=\left[B^{N}\right]^{T}[V]$

Denoting as $\left[\Phi^{N}\right]$ the $(n \times N)$ real matrix defined by the column truncation of matrix $[\Phi]$ with respect to the $N$ largest singular values, we then have

$\left[\Phi^{N}\right]=\left[B^{N}\right]\left[\Psi^{N}\right]$.

In this equation, $\left[\Psi^{N}\right]$ is the eigenvector matrix solution of the eigenvalue problem

$\left[\mathcal{A}^{N}\right]\left[\Psi^{N}\right]=\left[\Psi^{N}\right]\left[\Lambda^{N}\right]$,

where $\left[\Lambda^{N}\right]$ is the $(N \times N)$ real diagonal matrix defined as the truncation of matrix $[\Lambda]$ with respect to the $N$ largest singular values. 
3.3 Construction of the mean nonlinear reduced-order computational model

The mean nonlinear reduced-order computational model is explicitly constructed from the knowledge of the POD basis. The construction is carried out in the context of the threedimensional finite element method. The finite elements used here are isoparametric solid finite elements with 8 nodes and the numerical integration is carried out with $r$ Gauss integration points.

Let $[D]$ be the $(6 \times 6)$ real matrix which represents the usual Hooke matrix related to the fourth-order elasticity tensor. For a given isoparametric finite element, the displacement field $\widetilde{\mathbf{u}}(\mathbf{y})$ with $\mathbf{y} \in[-1,1]^{3}$, is defined by

$\widetilde{\mathbf{u}}(\mathbf{y})=[N(\mathbf{y})] \widetilde{\mathbb{u}}$,

in which the $(3 \times 24)$ real matrix $[N(\mathbf{y})]$ defines the interpolation functions and where the $\mathbb{R}^{24}$-vector $\widetilde{u}$ is made up of the degrees-of-freedom of the finite element. Let $\mathcal{I}$ be the set of indices defined by $\mathcal{I}=\{(i, j) \in\{(11),(22),(33),(12)$, (13), (23) $\}\}$ and corresponding with the set $\mathfrak{J}=\{1,2,3,4$, $5,6\}$. From Eq. (32), it can be deduced that

$$
\begin{aligned}
& \varepsilon_{i j}(\widetilde{\mathbf{u}})\left(1-\delta_{i j}\right)+\varepsilon_{j i}(\widetilde{\mathbf{u}})=[B(\mathbf{y})]_{J k} \widetilde{\mathbb{U}}_{k}, \\
& (i, j) \in \mathcal{I}, J \in \mathfrak{J}, k=\{1, \ldots, 24\},
\end{aligned}
$$

in which $[B(\mathbf{y})]$ is the $(6 \times 24)$ real matrix whose components are obtained by the calculation of partial derivatives of the interpolation functions contained in the matrix $[N(\mathbf{y})]$.

The first step consists in calculating, for each finite element, the elementary contributions of the linear, quadratic and cubic internal forces projected on the POD basis. Then, for a given finite element, the $\mathbb{R}^{24}$-vector, constituted of the internal forces induced by the POD basis vector $\varphi^{\beta}$ and related to the linear stiffness term, is written as

$\widetilde{\mathfrak{F}}^{(1)}\left(\widetilde{\boldsymbol{\varphi}}^{\beta}\right)=\sum_{i=1}^{r}\left[B\left(\mathbf{y}_{i}\right)\right]^{T}[D]\left[B\left(\mathbf{y}_{i}\right)\right] \widetilde{\boldsymbol{\varphi}}^{\beta}(\operatorname{det} J) w_{i}$,

in which $\widetilde{\varphi}^{\beta}$ is the spatial restriction of POD basis vector $\varphi^{\beta}$ to the considered finite element. Further, $\mathbf{y}_{i}, i=\{1, \ldots, r\}$ are the locations of the $r$ Gauss integration points related to the isoparametric finite element with $w_{i}$ the corresponding weights. Finally, $(\operatorname{det} J)$ is the Jacobian of the transformation. Let $\left[C_{k}(\mathbf{y})\right]$ be the $(3 \times 24)$ real matrix defined by

$\widetilde{u}_{s, l}(\mathbf{y}) \widetilde{u}_{s, m}(\mathbf{y})=\widetilde{u}^{T}\left[C_{l}(\mathbf{y})\right]^{T}\left[C_{m}(\mathbf{y})\right] \widetilde{u}$.

We then introduce the real $(6 \times 24)$ matrix $\left[E_{\beta}(\mathbf{y})\right]$ whose row number $J \in \mathfrak{J}$ is defined by

$\widetilde{\varphi}^{\beta, T}\left(\left[C_{i}(\mathbf{y})\right]^{T}\left[C_{j}(\mathbf{y})\right]\left(1-\delta_{i j}\right)+\left[C_{j}(\mathbf{y})\right]^{T}\left[C_{i}(\mathbf{y})\right]\right)$.

Then, for a given finite element, the $\mathbb{R}^{24}$-vector constituted of the internal forces induced by the POD basis vectors $\varphi^{\beta}$ and $\varphi^{\gamma}$, related to the first contribution of the quadratic stiffness term, is written as

$$
\begin{aligned}
& \widehat{\widetilde{\mathbb{F}}}^{(2)}\left(\widetilde{\boldsymbol{\varphi}}^{\beta}, \widetilde{\varphi}^{\gamma}\right)= \\
& \sum_{i=1}^{r}\left[B\left(\mathbf{y}_{i}\right)\right]^{T}[D]\left[E_{\beta}\left(\mathbf{y}_{i}\right)\right] \widetilde{\varphi}^{\gamma}(\operatorname{det} J) w_{i} .
\end{aligned}
$$

In the same way, the $\mathbb{R}^{24}$-vector, constituted of the internal forces induced by the POD basis vectors $\varphi^{\beta}, \varphi^{\gamma}$ and $\varphi^{\delta}$ and related to the cubic stiffness term, is written as

$$
\begin{aligned}
& \widetilde{\mathbb{F}}^{(3)}\left(\widetilde{\varphi}^{\beta}, \widetilde{\varphi}^{\gamma}, \widetilde{\varphi}^{\delta}\right)= \\
& \sum_{i=1}^{r}\left[E_{\beta}\left(\mathbf{y}_{i}\right)\right]^{T}[D]\left[E_{\gamma}\left(\mathbf{y}_{i}\right)\right] \widetilde{\varphi}^{\delta}(\operatorname{det} J) w_{i} .
\end{aligned}
$$

In a second step, for each type of stiffness, we proceed with the assembly of each of these elementary contributions. We then denote by $\mathbb{f}^{(1)}\left(\varphi^{\beta}\right), \widehat{\mathbb{f}}^{(2)}\left(\varphi^{\beta}, \varphi^{\gamma}\right)$ and $\mathbb{E}^{(3)}\left(\varphi^{\beta}, \varphi^{\gamma}, \varphi^{\delta}\right)$ the $\mathbb{R}^{n}$-vectors of these internal loads. The mean nonlinear reduced-order computational model is then described by

$$
\begin{aligned}
\mathcal{K}_{\alpha \beta}^{(1)} & =\varphi^{\alpha, T} \mathbb{E}^{(1)}\left(\varphi^{\beta}\right), \\
\hat{\mathcal{K}}_{\alpha \beta \gamma}^{(2)} & =\varphi^{\alpha, T} \widehat{\mathbb{F}}^{(2)}\left(\varphi^{\beta}, \varphi^{\gamma}\right), \\
\mathcal{K}_{\alpha \beta \gamma \delta}^{(3)} & =\varphi^{\alpha, T} \mathbb{E}^{(3)}\left(\varphi^{\beta}, \varphi^{\gamma}, \varphi^{\delta}\right) .
\end{aligned}
$$

The quadratic stiffness tensor $\mathcal{K}_{\alpha \beta \gamma}^{(2)}$ of the mean reduced nonlinear computational model is then build from Eq. (16). It should be noted that the $\mathcal{K}_{\alpha \beta}^{(1)}, \widehat{\mathcal{K}}_{\alpha \beta \gamma}^{(2)}$ and $\mathcal{K}_{\alpha \beta \gamma \delta}^{(3)}$ contributions have to be explicitly known for constructing the stochastic nonlinear reduced-order computational model in the general case of complex structures.

\section{Nonparametric stochastic modeling of uncertainties}

This Section concerns the construction of the stochastic nonlinear computational model. It is assumed that the mean computational model contains both system parameter uncertainties and model uncertainties which will be represented by the nonparametric probabilistic approach. The nonparametric probabilistic approach is necessarily implemented from a mean reduced computational model, which is chosen to be the mean reduced-order nonlinear model described in the above Section.

\subsection{Definition of a reshaped stiffness matrix}

The main idea of the nonparametric probabilistic approach is to replace each of the matrices of a given mean reduced computational model by a random matrix whose probability model is constructed from the maximum entropy principle using the available information $[17,19]$. In the usual 
linear case, the random matrices issued from the mechanical system are with values in the set of the symmetric positivedefinite matrices. In the present geometrical nonlinear context, the nonlinear equations involve nonlinear operators. In this case, we then introduce [9] the $(P \times P), P=N(N+1)$ matrix $[\mathcal{K}]$ as the real defined by

$[\mathcal{K}]=\left[\begin{array}{cc}{\left[\mathcal{K}^{(1)}\right]} & {\left[\widehat{\mathcal{K}}^{(2)}\right]} \\ {\left[\widehat{\mathcal{K}}^{(2)}\right]^{T}} & 2\left[\mathcal{K}^{(3)}\right]\end{array}\right]$.

In Eq. (42), $\left[\widehat{\mathcal{K}}^{(2)}\right]$ and $\left[\mathcal{K}^{(3)}\right]$ are respectively the $\left(N \times N^{2}\right)$ and $\left(N^{2} \times N^{2}\right)$ real matrices resulting from the following reshaping operation

$$
\begin{aligned}
& {\left[\widehat{\mathcal{K}}^{(2)}\right]_{\alpha J}=\widehat{\mathcal{K}}_{\alpha \beta \gamma}^{(2)} \quad, \quad \text { with } J=(\beta-1) N+\gamma} \\
& {\left[\mathcal{K}^{(3)}\right]_{I J}=\mathcal{K}_{\alpha \beta \gamma \delta}^{(3)},} \\
& \quad \text { with } I=(\alpha-1) N+\beta \text { and } J=(\gamma-1) N+\delta
\end{aligned}
$$

The key point consists in showing that the matrix $[\mathcal{K}]$ is a symmetric and positive-definite matrix [9]. Consequently, the nonparametric probabilistic approach initially introduced in the linear context can easily be extended to the geometrically nonlinear context.

\subsection{Construction of the random matrix model}

The mean reduced matrix $[\mathcal{K}]$ is then replaced by the random matrix $[\mathcal{K}]$ such that $\mathcal{E}\{[\mathcal{K}]\}=[\mathcal{K}]$ in which $\mathcal{E}$ is the mathematical expectation. The random matrix $[\mathcal{K}]$ is then written as $[\mathcal{K}]=\left[L_{K}\right]^{T}\left[\mathbf{G}_{K}\right]\left[L_{K}\right]$ in which $\left[L_{K}\right]$ is a $(P \times P)$ real upper matrix such that $[\mathcal{K}]=\left[L_{K}\right]^{T}\left[L_{K}\right]$. Further, $\left[\mathbf{G}_{K}\right]$ is a full random matrix with value in the set of all the positive-definite symmetric $(P \times P)$ matrices. The probability model of the random matrix $\left[\mathbf{G}_{K}\right]$ is constructed from the maximum entropy principle with the available information. All details concerning the construction of this probability model can be found in $[17,18]$. The dispersion of the random matrix $\left[\mathbf{G}_{K}\right]$ is controlled by one real positive parameter $\delta \in \mathcal{D}$ called the dispersion parameter. In addition, there exists an algebraic representation of this random matrix useful to the Monte Carlo numerical simulation. From the random matrix $[\mathcal{K}]$ the random linear, quadratic and cubic stiffness terms $\mathcal{K}_{\alpha \beta}^{(1)}, \widehat{\mathcal{K}}_{\alpha \beta \gamma}^{(2)}$ and $\mathcal{K}_{\alpha \beta \gamma \delta}^{(3)}$ can easily be deduced from Eqs. (42)-(44). The random matrix model is then defined by

$\mathbf{U}=\left[\Phi^{N}\right] \mathbf{Q}$,

in which $\mathbf{Q}=\left(\mathbf{Q}_{1}, \ldots, \mathbf{Q}_{N}\right)$ is the $\mathbb{R}^{N}$-valued random satisfying for $\alpha=\{1, \ldots, N\}$ the equations

$\mathcal{K}_{\alpha \beta}^{(1)} \mathbf{Q}_{\beta}+\mathcal{K}_{\alpha \beta \gamma}^{(2)} \mathbf{Q}_{\beta} \mathbf{Q}_{\gamma}+\mathcal{K}_{\alpha \beta \gamma \delta}^{(3)} \mathbf{Q}_{\beta} \mathbf{Q}_{\gamma} \mathbf{Q}_{\delta}=\mathcal{F}_{\alpha}$, with

$\mathcal{K}_{\alpha \beta \gamma}^{(2)}=\frac{1}{2}\left(\widehat{\mathcal{K}}_{\alpha \beta \gamma}^{(2)}+\widehat{\mathcal{K}}_{\beta \gamma \alpha}^{(2)}+\widehat{\mathcal{K}}_{\gamma \alpha \beta}^{(2)}\right)$.

It should be noted that the nonlinear dependency of the random linear, quadratic and cubic stiffness terms with respect to dispersion parameter $\delta$ has been omitted in Eq.(46) for clarity of presentation.

\section{Identification of the stochastic nonlinear reduced-order computational model}

This Section is devoted to the identification of the parameter $\delta$ in $\mathcal{D}$ of the stochastic nonlinear reduced-order computational model using simulated experiments.

It is assumed that a collection of $n_{\text {exp }}$ simulated experiments are available at $n_{\text {obs }}$ spatial locations. Denote by $U_{j}^{e x p}\left(s, \theta_{k}\right)$ the $k t h$ simulated experiment at DOF number $j$ as a function of the load increment $s$. The corresponding observation calculated with the stochastic nonlinear reducedorder computational model is denoted by $U_{j}(\delta, s)$ and is a function of the parameter $\delta$ which has to be identified. Let $U_{j}^{+}(\delta, s)$ (resp. $\left.U_{j}^{-}(\delta, s)\right)$ and $U_{j}^{e x p,+}(s)\left(\operatorname{resp} . U_{j}^{e x p,-}(s)\right.$ ) be the upper- (resp. lower-) envelope of the confidence region of observation $U_{j}(\delta, s)$ obtained with a probability level 0.98 and the upper- (resp. lower-) envelope of experiments $U_{j}^{\exp }(s)$.

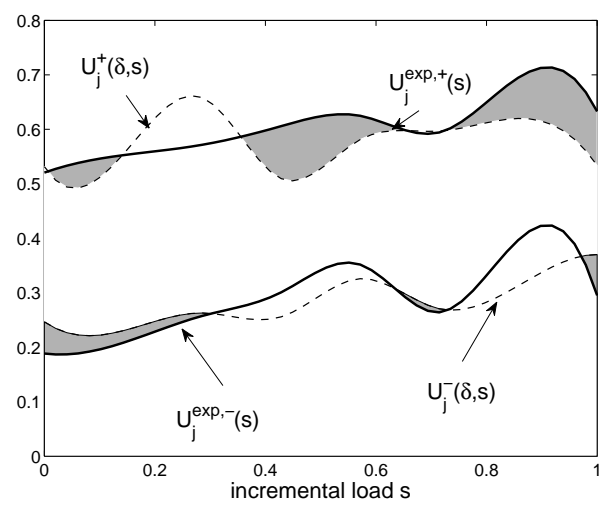

Fig. 2 Definition of the cost function

Then, a cost function $j(\delta)$ is introduced to quantify the departure of the simulated experiments from the confidence region constructed with the stochastic reduced-order nonlinear computational model. Penalty terms are introduced only in the regions for which the simulated experiments are not within the confidence region constructed with the stochastic nonlinear reduced-order computational model (see Fig. 2). The cost function is then a positive decreasing function of parameter $\delta$. Indeed, the cost function is equal to zero as soon as the simulated experiments belong to the confidence 
region of the random observation. Specifically, it is proposed here to define the cost function $j(\delta)$ to be minimized as

$j(\delta)=\left\|\boldsymbol{\Delta}^{+}(\delta, \cdot)\right\|_{\mathbb{B}}^{2}+\left\|\boldsymbol{\Delta}^{-}(\delta, \cdot)\right\|_{\mathbb{B}}^{2}$.

In this equation, $\|\cdot\|_{\mathbb{B}}$ is the $L^{2}$ norm over the load incremental band $\mathbb{B}=[0,1]$ and where $\boldsymbol{\Delta}^{+}(\delta, s)$ and $\boldsymbol{\Delta}^{-}(\delta, s)$ are the $\mathbb{R}^{n_{o b s}}$-vectors whose component $j$ is defined by

$$
\begin{aligned}
\Delta_{j}^{+}(\delta, s)= & \left\{U_{j}^{+}(\delta, s)-U_{j}^{\exp ,+}(s)\right\} \times \\
& \left\{1-H\left(U_{j}^{+}(\delta, s)-U_{j}^{e x p,+}(s)\right)\right\}, \\
\Delta_{j}^{-}(\delta, s)= & \left\{U_{j}^{-}(\delta, s)-U_{j}^{\exp ,-}(s)\right\} \times \\
& \left.\left\{H\left(U_{j}^{-}(\delta, s)-U_{j}^{\exp ,+}(s)\right)\right)\right\} .
\end{aligned}
$$

Finally, $x \mapsto H(x)$ is the Heaviside function. The identification of $\delta$ consists in solving the optimization problem

$\delta^{\text {opt }}=\arg \min _{\delta \in \mathcal{D}} j(\delta)$.

\section{Numerical application}

The objective of this application is to show the efficiency of the presented methodology. The application is a threedimensional linear elastostatic problem in the geometrically nonlinear context. The material is chosen to be homogeneous and isotropic. The extension to the nonhomogeneous case and to the anisotropic case is straightforward. A preliminary calculation is carried out with MD NASTRAN in order to get the reference solution from which the POD basis is calculated. The stochastic nonlinear reduced-order computational model is then constructed as a function of identification parameter $\delta$.

Note that the simulated experiments have been obtained by numerical simulations for a family of structures around the mean structure. Specifically, the geometrical characteristics of each structure of the family are modified with respect to those of the mean structure. Moreover, the material characteristics of these structures are inhomogenous at the contrary of the mean structure. Consequently, the mean computational model can never reproduce the simulated experiments which justifies the use of a stochastic nonlinear reduced-order computational model.

\subsection{Mean finite element model}

The three-dimensional bounded domain $\Omega$, called the curved structure, results from the geometrical transformation of a slender rectangular domain $\Omega^{\prime}$ into a curved domain $\Omega$. The slender domain $\Omega^{\prime}$ is defined in a Cartesian system $\left(0, \mathbf{e}_{1}, \mathbf{e}_{2}, \mathbf{e}_{3}\right)$ such that $\Omega^{\prime}=\{] 0, l[\times] 0, b[\times] 0, h[\}$ with $l=10 \mathrm{~m}, b=1 \mathrm{~m}, \mathrm{~h}=1.5 \mathrm{~m}$. The curved structure $\Omega$ is then defined as a part of a cylindrical domain such that
$\Omega=\{] r_{i}, r_{e}[\times] 0, \alpha[\times] 0, h[\}$ in a local cylindrical system defined as $\left(0, \mathbf{e}_{r}, \mathbf{e}_{\theta}, \mathbf{e}_{3}\right)$ with $\alpha=\frac{35 \pi}{18}, r_{i}=\frac{l-b \alpha}{\alpha}$ and $r_{e}=\frac{l}{\alpha}$. Let $\Gamma_{0} \subset \partial \Omega$ be the boundary described as $\left\{r \in\left[r_{i}, r_{e}\right], \theta=-\frac{\pi}{2}, x_{3} \in[0, h]\right\}$. The structure is assumed to be fixed on this boundary so that we have a Dirichlet condition on $\Gamma_{0}$. The structure is free on boundary $\partial \Omega \backslash \Gamma_{0}$. The structure is subjected to external surface loads applied along both directions $\mathbf{e}_{1}$ and $\mathbf{e}_{2}$ in the end section defined by $\left\{r \in\left[r_{i}, r_{e}\right], \theta=\frac{13 \pi}{9}, x_{3} \in[0, h]\right\}$. The Young modulus and the Poisson coefficient of the homogeneous and isotropic linear elastic material are $E=10^{10} \mathrm{~N} . \mathrm{m}^{-2}$ and $\nu=0.15$. The finite element model is a regular mesh of 102425 nodes and $240 \times 16 \times 24=92160$ finite elements constituted of 8-nodes solid elements with $r=8$ Gauss integration points. Therefore, the mean computational model has $n=306123$ degrees of freedom (see Fig. 3).

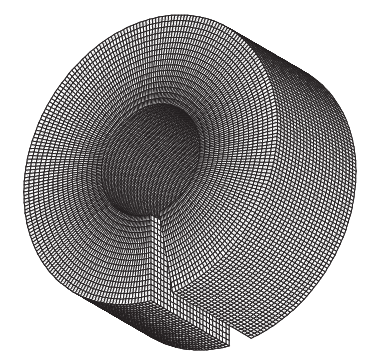

Fig. 3 Finite element model

The discretization of the external loads yields point loads applied to the nodes of the end section along the direction $\mathbf{e}_{2}$ with intensity $1,333,333 \mathrm{~N}$ and yields point loads applied to the nodes of the end section along the direction $\mathbf{e}_{1}$ with intensity $-166,666 N$. An initial imperfection with a maximum amplitude of $200 \mu \mathrm{m}$ is added to the initial structure in order to construct the mean nonlinear computational model. This initial imperfection is defined by the first linear elastic buckling mode of the curved structure $\Omega$ whose shape is zoomed and shown in Fig. 4. In the present case, the first linear elastic buckling mode is a bending mode with eigenvalue $\lambda_{c}=0.1825$ corresponding to a critical load $243,333 \mathrm{~N}$ along the direction $\mathbf{e}_{2}$ (respectively $-30,415 \mathrm{~N}$ along the direction $\mathbf{e}_{1}$ ).

In order to simulate the post-buckling mechanical response, the static nonlinear calculations are carried out by solving Eq. (24) using MD NASTRAN with an algorithm based on the arc-length method. The displacement field is calculated using $n_{t}=110$ load increments. Fig. 5 shows the deformed curved structure. 


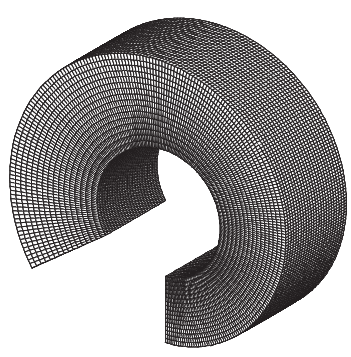

Fig. 4 Shape of the first linear elastic buckling mode

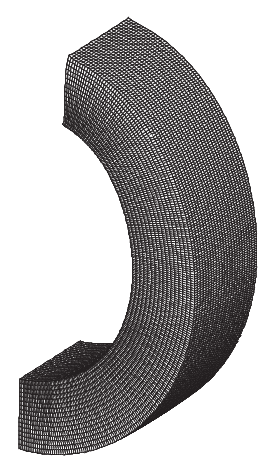

Fig. 5 Deformed curved structure

\subsection{Construction of the experimental data basis}

In this numerical application, $n_{\exp }=8$ simulated experiments related to the static nonlinear response are calculated. The simulated experiments are observed at $n_{\text {obs }}=$ 2 observation points corresponding to the DOFs located at the middle of the end section in the directions $\left(0, \mathbf{e}_{1}\right)$ and $\left(0, \mathbf{e}_{2}\right)$. The simulated static nonlinear responses are denoted by $u_{o b s}^{\text {exp }, k}(s)$, for $k$ in $\left\{1, \ldots, n_{\text {exp }}\right\}$. The corresponding quantity defined for the mean nonlinear computational model is denoted by $u_{o b s}(s)$. The simulated experiments are generated as follows. The geometrical parameters $l, b$ and $h$ are replaced by the random uniform variables $L, B$ and $H$ centered around geometrical parameters $l, b$ and $h$ with supports $[0.95 l, 1.05 l],[0.95 b, 1.05 b]$ and $[0.95 h, 1.05 h]$. Moreover, it is assumed that the Young modulus is inhomogeneous with $10 \%$ of variation around its mean value. This is achieved by replacing the deterministic value $E$ by a stochastic field $E(\mathbf{x})$ which is defined by

$E(\mathbf{x})=E+\sum_{j=1}^{J} \xi_{j} \mathbf{b}_{j}(\mathbf{x})$

in which $\xi_{1}, \ldots, \xi_{J}$ are independent uniform random variables with zero mean and standard deviation $\sigma=0.1 E / \sqrt{3}$ and where the functions $\mathbf{b}_{j}(\mathbf{x})$ are given smooth functions. For convenience, these smooth functions are taken as the spatial average over each element of the eigenvectors associated with the $J$ lowest eigenvalues of the usual generalized eigenvalue problem related to the linear dynamics.

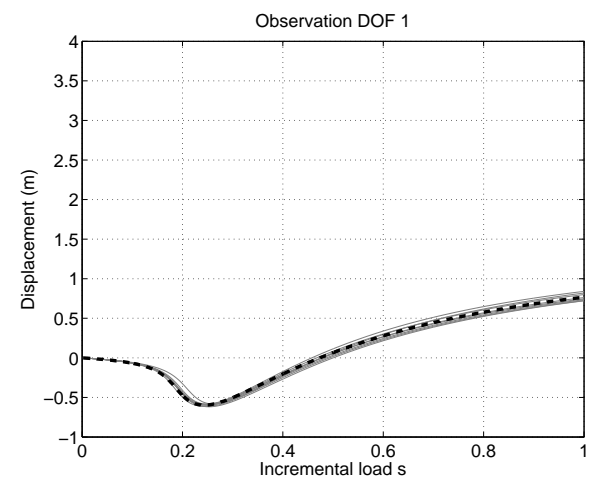

Fig. 6 Displacement response at observation DOF 1 as a function of the incremental load $s$ : mean computational model (thick dashed line), simulated experiments (thin gray lines)

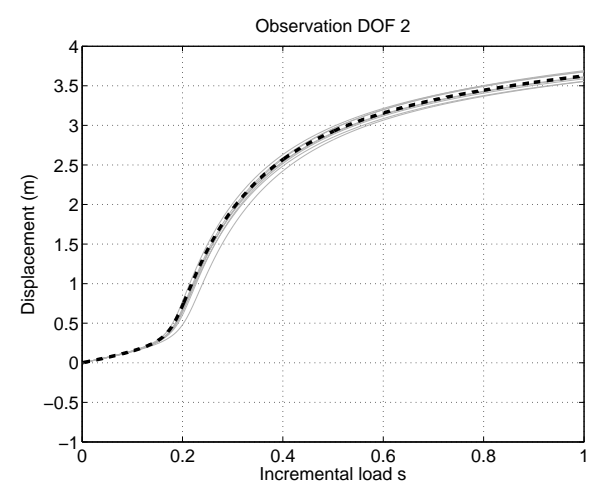

Fig. 7 Displacement response of observation DOF 2 as a function of the incremental load $s$ : mean computational model (thick dashed line), simulated experiments (thin gray lines)

Figures 6 and 7 compare the static nonlinear responses as a function of the incremental load $s$ for both mean nonlinear computational model and simulated experiments. Since the simulated experiments are scattered around the response calculated with the mean nonlinear computational model, it can be deduced that the use of a stochastic nonlinear computational model is justified. 


\subsection{Construction of the POD basis}

The nonlinear response shown in Fig. 5 is then used for calculating the POD basis as described in Section 3.2. Let $\operatorname{Conv}_{\mathrm{POD}}(N)$ be the function defined by

$\operatorname{Conv}_{\mathrm{POD}}(N)=1-\frac{1}{\operatorname{tr}([A])} \sum_{j=1}^{N} \Lambda_{j}$

for which the calculation of $\operatorname{tr}([A])$ does not require the computation of matrix $[A]$. Fig. 8 shows the graph of the function $N \mapsto \operatorname{Conv}_{\mathrm{POD}}(N)$ in a logarithmic scale. It can be seen that a good convergence is obtained for $N=10$. From now on, all numerical calculations are carried out with $N=12$. The mean nonlinear reduced-order computational model is then constructed and solved using the Crisfield algorithm [3] based on the arc-length method.

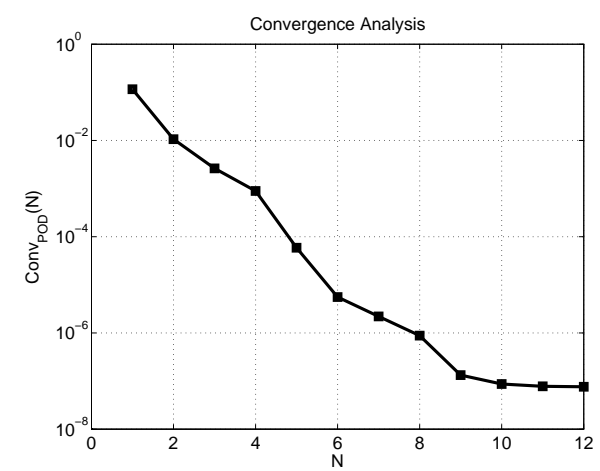

Fig. 8 Convergence analysis : graph of $N \mapsto \operatorname{Conv}_{\mathrm{POD}}(N)$.

6.4 Experimental identification of the stochastic nonlinear reduced-order computational model

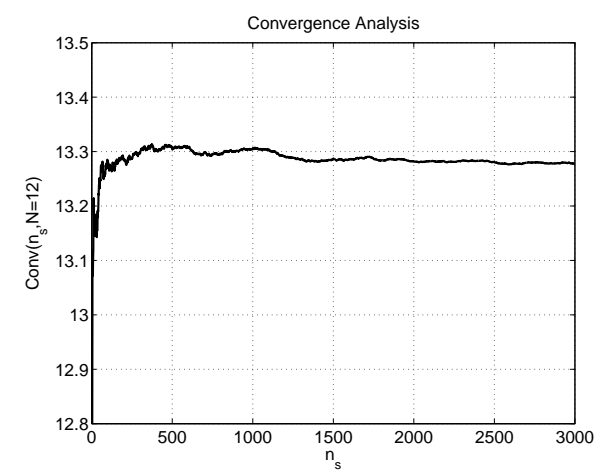

Fig. 9 Convergence analysis : graph of $n_{s} \mapsto \operatorname{Conv}\left(n_{s}\right)$.

The construction of the stochastic nonlinear reducedorder computational model using the nonparametric probabilistic approach is performed as explained in Section 4. A stochastic convergence analysis is then carried out to define the number $n_{s}$ of Monte Carlo realizations to be kept in the numerical simulation. Let $n_{s} \mapsto \operatorname{Conv}\left(n_{s}\right)$ be the function defined by

$\operatorname{Conv}\left(n_{s}\right)=\left\{\frac{1}{n_{s}} \sum_{j=1}^{n_{s}}\|\| \mathbf{U}\left(\theta_{j}\right) \mid \|^{2}\right\}^{1 / 2}$

in which $\left.\left.\left\|\mid \mathbf{U}\left(\theta_{j}\right)\right\|\left\|=\max _{s}\right\| \mathbf{U}\left(\theta_{j}, s\right)\right)\|,\| \mathbf{U}\left(\theta_{j}, s\right)\right) \|^{2}=$ $\sum_{k=1}^{n} U_{k}^{2}\left(\theta_{j}, s\right)$ where $U_{k}\left(\theta_{j}, s\right)$ is the $j t h$ realization of the random response at DOF $k$ for a given load increment $s$. Figure 9 displays the graph $n_{s} \mapsto \operatorname{Conv}\left(n_{s}\right)$ obtained with a dispersion parameter $\delta=0.6$. Convergence is reached for $n_{s}=1500$. The identification of $\delta$ is then carried out by constructing the non-differentiable cost function $\delta \mapsto j(\delta)$ using the Monte Carlo numerical simulation. The cost function is a positive decreasing function of parameter $\delta$. As soon as the simulated experiments belong to the confidence region of the random observation, the cost function is equal to zero.

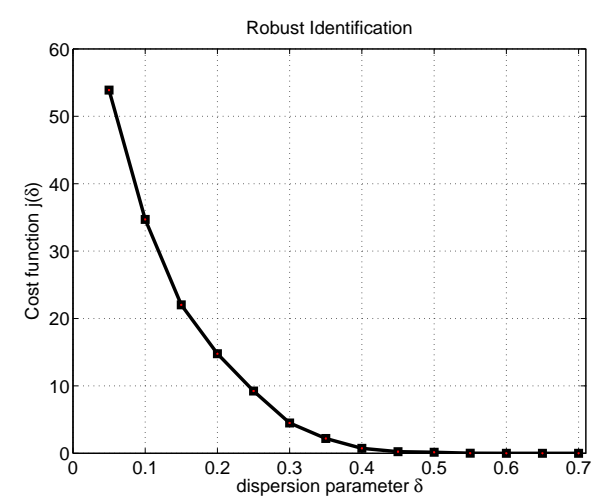

Fig. 10 Robust identification : graph of $\delta \mapsto j(\delta)$.

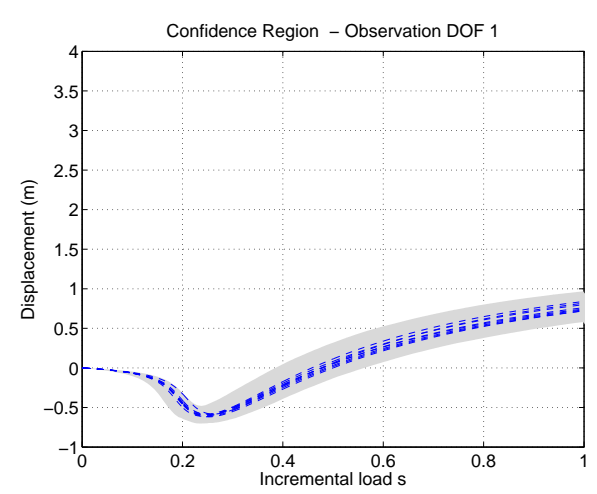

Fig. 11 Robust identification for observation DOF 1 : graph of the experimental data $s \mapsto u_{o b s}^{e x p, k}(s)$ (thin dashed lines), graph of the confidence region of the random response $s \mapsto U_{o b s}(s)$ (grey region). 


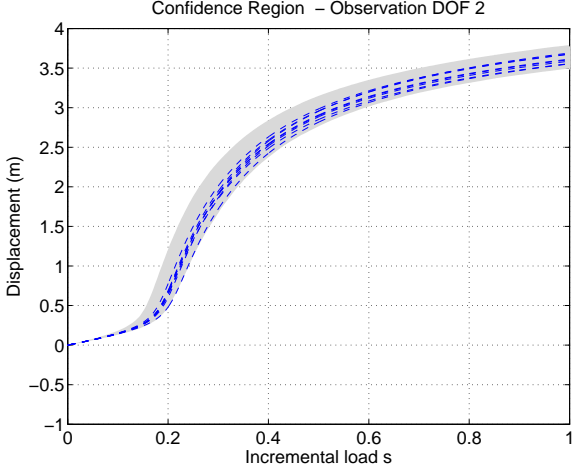

Fig. 12 Robust identification for observation DOF 2: graph of the experimental data $s \mapsto u_{o b s}^{e x p, k}(s)$ (thin dashed lines),graph of the confidence region of the random response $s \mapsto U_{o b s}(s)$ (grey region).

Figure 10 shows the graph of the cost function $\delta \mapsto j(\delta)$. It can be seen that the optimal value is given by $\delta^{\text {opt }}=$ 0.55 . Figures 11 and 12 display the graph of the confidence region of the optimal random response $U_{o b s}\left(\delta^{o p t}, s\right)$ as a function of the load increment $s$ for both observations. It then can be seen that there is a good agreement between the optimal stochastic nonlinear reduced-order computational model and the simulated experiments.

\section{Conclusion}

In the present paper, a methodology has been proposed for constructing a stochastic nonlinear reduced-order computational model for any three-dimensional structure with geometric nonlinearities and linear constitutive equation. The mean nonlinear reduced-order computational model is constructed by projection on the POD basis obtained from the mean nonlinear computational model. All the integrals involved in the weak formulation after projection on the POD basis are explicitly estimated using three-dimensional solid finite elements. The direct evaluation of the stiffness parameters of the mean nonlinear reduced-order model proposed and accomplished here guarantees the necessary properties (e.g. positive definiteness) of the model. It is further achievable for any three-dimensional finite element mean computational model. An application is presented and a methodology to perform the identification of the stochastic nonlinear reduced-order computational model using simulated experiments is proposed. If experimental data is available, the simulated experiments are then replaced by such data.

\section{References}

1. Capiez-Lernout, E., Soize, C.: Robust design optimization in computational mechanics. ASME Journal of Applied Mechanics 75(2), 021,001 1-021,001 11 (2008)
2. Capiez-Lernout, E., Soize, C.: Robust updating of uncertain damping models in structural dynamics for low- and medium-frequency ranges. Mechanical Systems and Signal Processing 22(8), 17741792 (2008)

3. Crisfield, M.: Non-linear finite element analysis of solids and structures, Vol. 1 : essentials. John Wiley and Sons, Chichester (1997)

4. Hodges, D., Shang, X., Cesnik, C.: Finite element solution of nonlinear intrinsic equations for curved composite beams. Journal of the American Helicopter Society 41(4), 313-321 (1996)

5. Hollkamp, J.J., Gordon, R.W.: Reduced-order models for nonlinear response prediction: Implicit condensation and expansion. Journal of Sound and Vibration 318(4-5), 1139-1153 (2008)

6. Huang, H., Han, Q.: Research on nonlinear postbuckling of functionally graded cylindrical shells under radial loads. Computers \& Structures 92(6), 1352-1357 (2010)

7. Lee, S.H.: MSC/Nastran Handbook for nonlinear analysis, version 67. Lee, S.-H. editor (1992)

8. Lindgaard, E., Lund, E., Rasmussen, K.: Nonlinear buckling optimization of composite structures considering "worst" shape imperfections. International Journal of Solids and Structures 47(2223), 3186-3202 (2010)

9. Mignolet, M.P., Soize, C.: Stochastic reduced order models for uncertain geometrically nonlinear dynamical systems. Computer Methods in Applied Mechanics and Engineering 197, 3951-3963 (2008)

10. Murthy, R., Wang, X., Mignolet, M.P.: Uncertainty-based experimental validation of nonlinear reduced order models. In: Proceedings of the RASD 2010, Southampton, 12-14 July 2010 (2010)

11. Muryavov, A., Rizzi, S.: Determination of nonlinear stiffness with application to random vibration of geometrically nonlinear structures. Computers \& Structures 81, 1513-1523 (2003)

12. Pai, P., Nayfeh, A.: A Fully Nonlinear-Theory of Curved And Twisted Composite Rotor Blades Accounting For Warpings and 3-Dimensional Stress Effects. International Journal of Solids and Structures 31(9), 1309-1340 (1994)

13. Sampaio, R., Soize, C.: Remarks on the efficiency of pod for model reduction in non-linear dynamics of continuous elastic systems. International Journal for Numerical Methods in Engineering 72(1), 22-45 (2007)

14. Schenk, C., Schuëller, G.: Buckling analysis of cylindrical shells with random geometric imperfections. International Journal of Nonlinear Mechanics 38(7), 1119-1132 (2003)

15. Schenk, C., Schuëller, G.: Buckling analysis of cylindrical shells with cutouts including random boundary and geometric imperfections. Computer Methods in Applied Mechanics and Engineering 196(35-36), 3424-3434 (2007)

16. Sirovich, L.: Turbulence and the dynamics of coherent structures . Quarterly of Applied Mathematics 45(3), 561-571 (1987)

17. Soize, C.: A nonparametric model of random uncertainties for reduced matrix models in structural dynamics. Probabilistic Engineering Mechanics 15(3), 277-294 (2000)

18. Soize, C.: A comprehensive overview of a non-parametric probabilistic approach of model uncertainties for predictive models in structural dynamics. Journal of Sound and Vibration 288(3), 623652 (2005)

19. Soize, C.: Random matrix theory for modeling random uncertainties in computational mechanics. Computer Methods in Applied Mechanics and Engineering 194(12-16), 1333-1366 (2005)

20. Soize, C., Capiez-Lernout, E., Durand, J.F., Fernandez, C., Gagliar dini, L.: Probabilistic model identification of uncertainties in computational models for dynamical systems and experimental validation. Computer Mechanical Methods in Applied Mechanics and Engineering 98(1), 150-163 (2008)

21. Tang, D., Dowell, E.: Nonlinear response of a non-rotating rotor blade to a periodic gust. Journal of Fluids and Structures 10(7), 721-742 (1996) 
22. Yvonnet, J., Zahrouni, H., Potier-Ferry, M.: A model reduction method for the post-buckling analysis of cellular microstructures. Computer Methods in Applied Mechanics and Engineering 197, 265-280 (2007) 